\title{
Ruptured Aneurysm in Sphenoid Sinus: Which Is the Best Treatment?
}

\author{
Gabriele Ronchetti ${ }^{\mathrm{a}}$ Pier Paolo Panciani ${ }^{\mathrm{a}, \mathrm{d}}$ \\ Claudio Cornali $^{\mathrm{a}}$ Dikran Mardighian $^{\mathrm{b}}$ \\ Andrea Bolzoni Villaret ${ }^{c}$ Roberto Stefini ${ }^{a}$ \\ Marco Maria Fontanella ${ }^{a}$ d Roberto Gasparotti ${ }^{b}$ \\ ${ }^{a}$ Neurosurgery and ${ }^{b}$ Neuroradiology Units, Departments of Neuroscience and \\ Diagnostic Imaging, and 'Department of Otorhinolaryngology, University of \\ Brescia, Brescia, and ${ }^{d}$ University of Turin, Turin, Italy
}

\section{Key Words}

Internal carotid artery aneurysm · Sphenoid sinus · Epistaxis · Coiling · Pipeline · Endoscopic wrapping

\begin{abstract}
Internal carotid artery (ICA) aneurysms involving the sphenoid sinus are uncommon, and their optimal treatment remains debated. We report the case of a patient presenting with recurrent epistaxis due to a bleeding cavernous ICA aneurysm. We suggest a combined endovascular and endoscopic approach when ICA occlusion may not be performed.
\end{abstract}

\section{Introduction}

Non-traumatic cavernous carotid aneurysms (CCA) are rare. They usually manifest with mass effect signs, as subarachnoid and extradural bleeding are uncommon. The lateral wall of the adjacent sphenoid sinus may be occasionally eroded by the aneurysm growth, and severe epistaxis may occur $[1,2]$. The hemodynamic features of such aneurysms are peculiar, their treatment is challenging and a definitive flow exclusion is often difficult to achieve.

We report the case of a patient with ruptured CCA presenting with recurrent epistaxis. Several endovascular procedures did not succeed for definitive treatment. We review and discuss the optimal management of these exceptional aneurysms. 


\section{Case Report}

On December 2011, an 81-year-old male was admitted to the ENT Department of his referral hospital for recurrent epistaxis from the right nostril, which had started 5 days earlier. His past medical history included mild diabetes and hypertension, with no history of local trauma or surgery.

Rhinoscopy and CT angiography demonstrated a right internal carotid artery (ICA) aneurysm extending into the sphenoid sinus ( $\underline{\mathbf{f i g} .1 \mathrm{1}}$ ). A nasal packing was applied, and the patient was referred to our hospital for treatment.

On admission, the neurological examination was normal. Cerebral angiography including 3D rotational digital subtraction angiography (3D-DSA) was performed, and the size and geometry of the aneurysm were accurately evaluated (fig. 1b). A right ICA occlusion test showed little cross-flow from the left, contraindicating the occlusion of the aneurysm parent vessel.

Open surgery was not considered because of the patient's age and location of the aneurysm. The placement of an autoexpandable intracranial stent was discarded due to the need of an antiplatelet therapy, considered risky at this stage. Once a sizable neck was identified, an endovascular embolization with coils alone was performed, with the aim to prevent further rebleeding. A complete exclusion of the aneurysm with patency of the carotid artery was obtained (fig. 1c). The postoperative period was uneventful, and the patient was discharged after 1 week without any symptoms.

Three days after the discharge, a new abundant epistaxis occurred. However, a selective angiography performed at the referral hospital did not show clear recanalization of the aneurysm.

A massive subsequent rebleeding caused obstruction of the airways, intubation and blood transfusion. The patient was referred again to our hospital, and a new DSA showed a partial recanalization of the neck with an overall aneurysm growth (ig. $2 \mathrm{a}$ ). A second endovascular coiling was attempted, resulting in an almost complete aneurysm exclusion (fig. 2b). The follow-up DSA performed 1 week later showed a new recanalization and further enlargement of the aneurysm (fig. 2c). After administering a 150-mg clopidogrel load and systemic heparinization, we attempted to direct a covered coronary stent into the carotid siphon, which was unsuccessful due to the proximal tortuosity of the vessel and the relative stiffness of the device. Therefore, a flow-diverter stent (Pipeline, ev3, Irvine, Calif., USA) was placed across the aneurysm neck (fig. 2d). The procedure was uneventful and the patient started a daily therapy with $75 \mathrm{mg}$ of clopidogrel. After $72 \mathrm{~h}$, a massive epistaxis recurred and the patient expired.

\section{Discussion}

The sphenoid sinus provides a 'free space' where CCA may enlarge in case of progressive bone erosion. When aneurysms bleed in this large low-pressure space, the complications of a standing blood clot formation may lead to recurrent and even fatal hemorrhages [3]. These patients need to be handled by a multidisciplinary team: a rare occurrence considering epistaxis from other sources that can be effectively managed by ENT doctors alone. Conservative management may be applied in the incidental cases; on the other hand, a treatment is advisable in ruptured aneurysms [1,4]. The present case and other reports in the literature suggest that therapeutic success can be hard to reach in such patients [5].

External neck compression, nasal packs and balloon occlusion may be used as temporary measures. The goal of definitive treatments is to exclude the aneurysm from the blood flow. Occlusion of the parent artery is effective and easy to achieve with both open and endovascular access, but in our and other cases it caused a high ischemic risk for the homolateral hemisphere. In our case, an extra-intracranial bypass was not 
indicated especially due to the patient's age and the time needed for verifying its patency.

The endovascular and less invasive procedures should be considered as first choice especially in the elderly, whereas surgical clipping is not favorable for the usual shape and size of these aneurysms and their location within the cavernous sinus [6].

In our experience, coiling appeared not adequate to ensure a definitive aneurysm exclusion. Similar results concerning ruptured CCA were reported by other authors [1]. We observed a continuous growth of the aneurysm despite several embolizations. We speculate that the high-flow blood displaced the coils, leading to aneurysm enlargement within the sphenoid sinus. One remote possibility to control recurrent bleeding episodes could be emergency endoscopic sinus surgery, performed immediately after the first endovascular procedure.

The dome of the aneurysm could be protected by both coiling and free muscular tissue graft, then blocked with cartilage and stored endoscopically into the sphenoid sinus. Experiences in skull base surgery and in cases of wrapped intracranial aneurysms show remarkable hemostatic effect of the muscle. This wrapping technique could reduce the pressure gradient across the aneurysm wall and stabilize the coils' position until the internal thrombus formation. Covered stents represent an effective tool, but the relevant limitations regarding navigation into the tortuous and stiff arteries of elderly patients should be carefully considered. Flow-diverter stents are another available option to assess. In our patient, the flow disruptive action was maybe not complete, probably due to the high pressure across the neck. This situation may mimic the presence of perforating vessels that usually remain patent when covered by the stent struts [7]. The use of stents is moreover limited in the early stages after bleeding because of the need of antiplatelet therapy.

\section{Conclusions}

The management of ruptured CCA is challenging. Parent artery occlusion cannot be safely performed in all cases, and surgical bypass may be considered in selected patients. Clipping is generally not recommended. Covered stents are difficult to be deployed; coiling and flow-diverter stents are unlikely to achieve complete aneurysm exclusion. We speculate that treatment of CCA with sphenoid sinus involvement should be based on ICA occlusion. When this is not feasible, in addition to endovascular coiling, sphenoid sinus storage with wrapping of the aneurysm should be attempted.

\section{Disclosure Statement}

The authors have no related conflicts to disclose. 

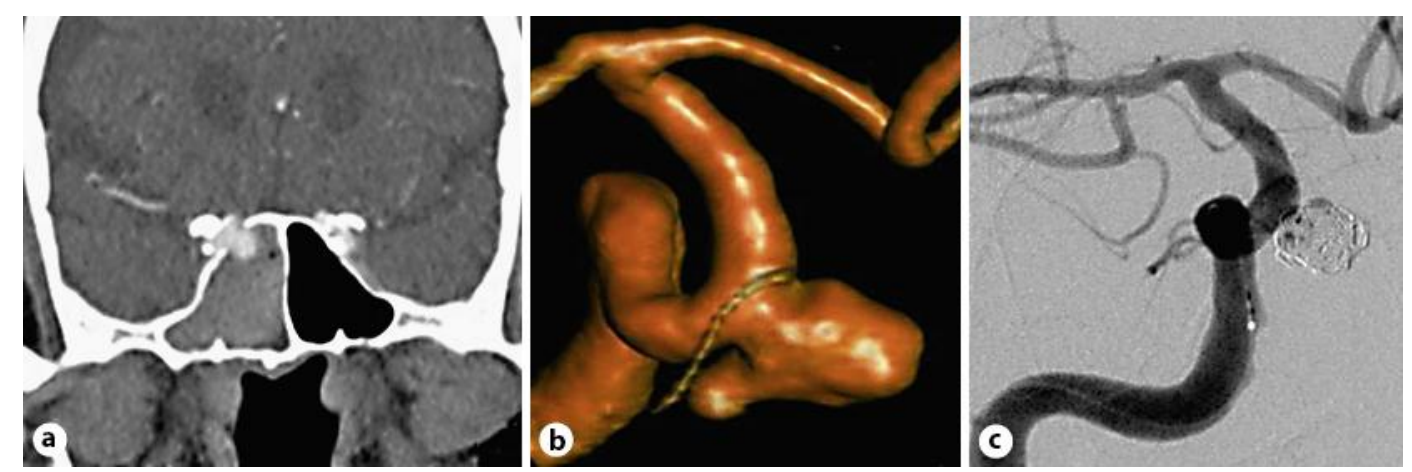

Fig. 1. a Contrast CT scan of the brain showing the right internal carotid aneurysm entering the sphenoid sinus. b Angiographic reconstruction of the $8 \times 7$-mm saccular aneurysm. c Complete aneurysm occlusion after coiling.
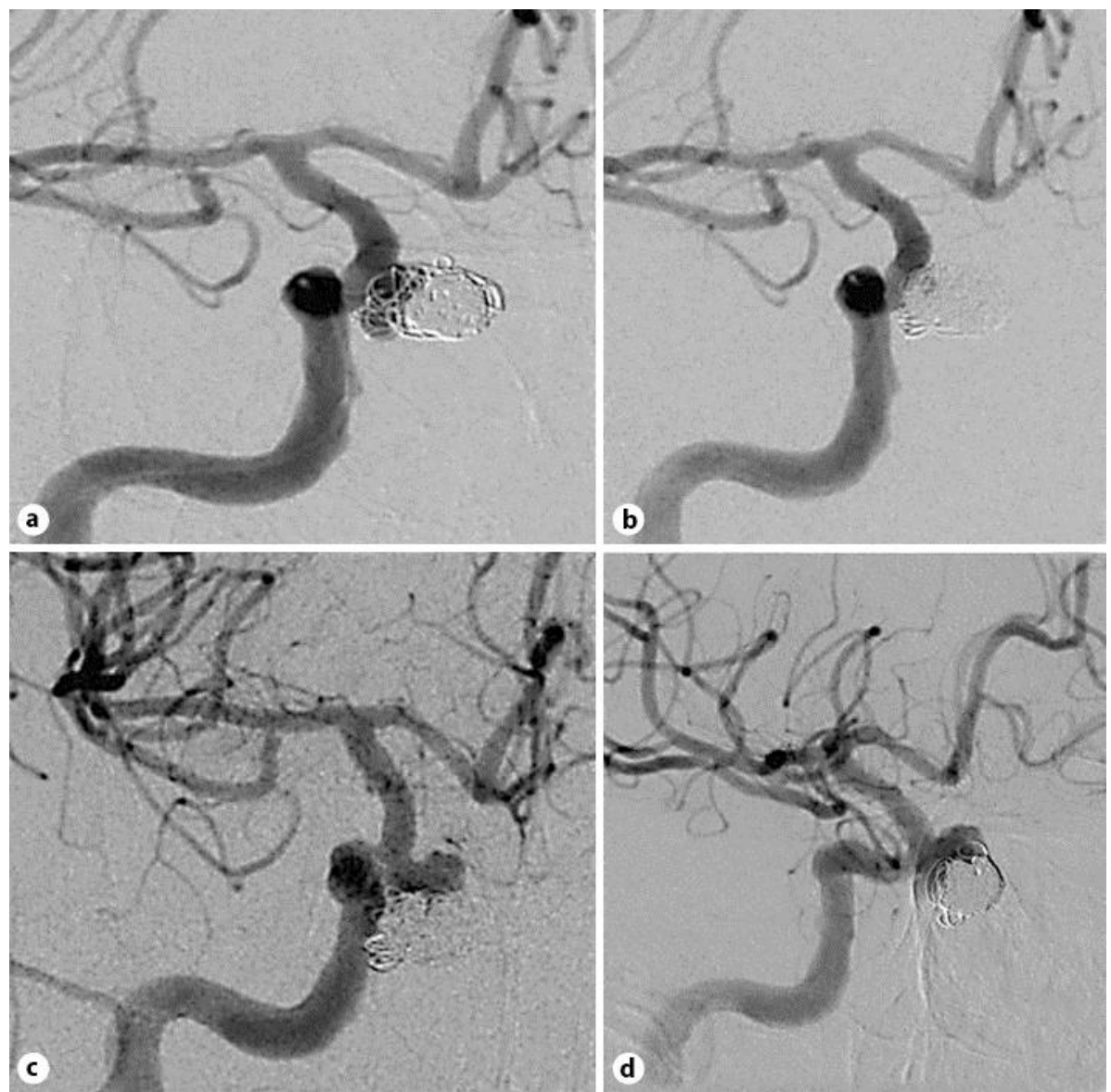

Fig. 2. Control angiography. a The aneurysm appears enlarged ( $11 \mathrm{~mm}$ in diameter) and the neck partly patent. b Nearly complete aneurysm occlusion after coiling. $\mathbf{c}$ Further aneurysm growth and recanalization despite two coiling procedures. $\mathbf{d}$ Flow-diverter stent positioning in the right ICA. 


\section{References}

1 Lehmann P, Saliou G, Page C, Balut A, Le Gars D, Vallée JN: Epistaxis revealing the rupture of a carotid aneurysm of the cavernous sinus extending into the sphenoid: treatment using an uncovered stent and coils. Review of literature. Eur Arch Otorhinolaryngol 2009;266:767-772.

2 Karkanevatos A, Karkos PD, Karagama YG, Foy P: Massive recurrent epistaxis from non-traumatic bilateral intracavernous carotid artery aneurysms. Eur Arch Otorhinolaryngol 2005;262:546-549.

-3 Urso-Baiarda F, Saravanappa N, Courteney-Harris R: An unusual cause of massive fatal epistaxis. Emerg Med J 2004;21:266.

-4 Liu JK, Gottfried ON, Amini A, Couldwell WT: Aneurysms of the petrous internal carotid artery: anatomy, origins, and treatment. Neurosurg Focus 2004;17:E13.

5 Singh H, Thomas J, Hoe WL, Sethi DS: Giant petrous carotid aneurysm: persistent epistaxis despite internal carotid artery ligation. J Laryngol Otol 2008;122:e18.

6 Garbossa D, Panciani PP, Fornaro R, Crobeddu E, Marengo N, Fronda C, et al: Subarachnoid hemorrhage in elderly: advantages of the endovascular treatment. Geriatr Gerontol Int 2012;12:46-49.

7 Kallmes DF, Ding YH, Dai D, Kadirvel R, Lewis DA, Cloft HJ: A new endoluminal, flow-disrupting device for treatment of saccular aneurysms. Stroke 2007;38:2346-2352. 